Pulsar Astronomy - 2000 and Beyond

ASP Conference Series, Vol. 202, 2000

M. Kramer, N. Wex, and R. Wielebinski, eds.

\title{
PuMa, the new Dutch PulsarMachine
}

\section{M.L.A. Kouwenhoven}

Sterrenkundig Instituut, Utrecht University, Postbox 80000, 3508 TA Utrecht, The Netherlands

P.C. van Haren, D. Driesens, J.J. Langerak, T.D. Beijaard

Instrumentele Groep Fysica, Utrecht University, Postbox 80000, 3508 TA Utrecht, The Netherlands

\section{J.L.L. Voûte, B.W. Stappers, R. Ramachandran}

Anton Pannekoek Instituut, University of Amsterdam, Kruislaan 403 , 1098 SJ Amsterdam, The Netherlands

\begin{abstract}
PuMa is a fully digital PulsarMachine the operation at the Westerbork Synthesis Radio Telescope (WSRT). Its design is based on the use of Digital Signal Processors. It has two modes of operation: a baseband recording mode and a digital filterbank mode.
\end{abstract}

\section{Design}

The signal path of PuMa is shown in figure 1. WSRT is a synthesis array telescope, which consists of 14 dishes, each with a diameter of 25 meter. The total area is equal to that of a 93 meter single dish. WSRT is equipped with multifrequency frontends, which are capable of observing over a large range of sky frequencies (see table in Kouwenhoven et al., these proceedings) and make simultaneous multifrequency observations possible. They deliver 8 bands of 10 $\mathrm{MHz}$ for each linear polarisation.

The voltages from the antennae are added together after correcting for the delay time between the dishes. The signal from each band is 12 bit sampled in Analog-to Digital Convertors (figure 2). These are industry standard video ADCs which sample the incoming data at $20 \mathrm{MHz}$ (Nyquist rate) with a temporal accuracy better than 5 ns. The total outgoing data rate is 2 polarizations $\times 8$ bands $\times 20 \mathrm{MHz}=320$ MSamples per second.

Each band is real time processed by four Digital Signal Processor Modules. These modules (figure 2) contain six SHARC DSPs. Each DSP can perform a

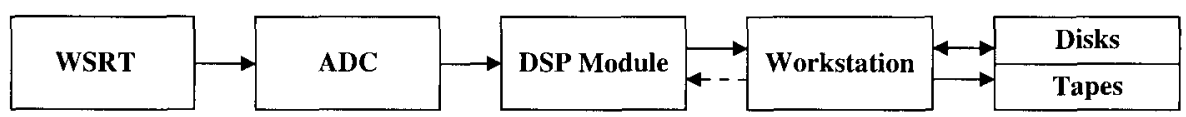

Figure 1. Signal path of PuMa. 

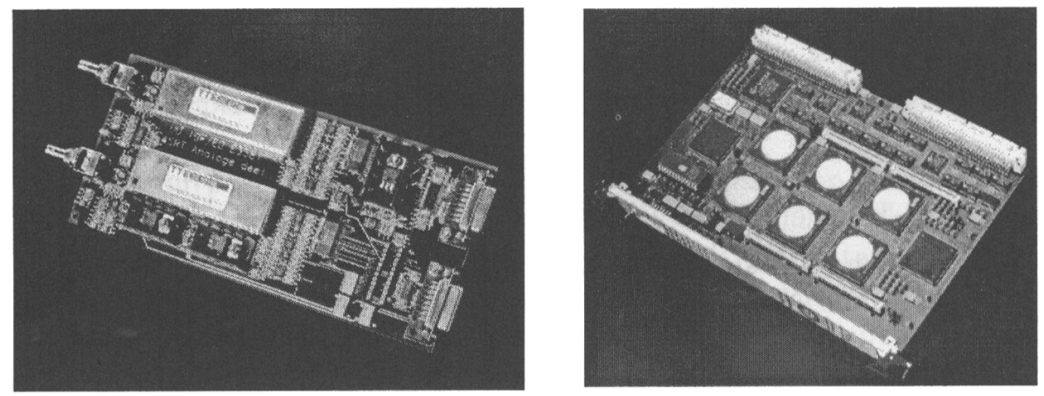

Figure 2. The Analog-to-Digital Convertors (left) and Digital Signal Processor module (right). The six DSPs are clearly visible as light circles.

2048 point complex Fast Fourier Transform within $1 \mathrm{~ms}$. The total computing power of the $8 \times 4 \times 6=192$ DSPs is more than 23 GFlop. The exact data reduction process depends on the mode of operation and the observation parameters. The data are written over a VME board in the shared memory of two HP743rt workstations, which control the DSPs. The maximum throughput rate is $20 \mathrm{MB} / \mathrm{s}$.

At the start of an observation the workstations load the software into the DSPs. They perform administration checks on the data coming from the DSPs and move them to eight Cheetah disks (9 GB capacity each). After the observation has finished the data are moved from the disks to two DDS-3 tape drives.

\section{Modes of operation}

We have developed two modes of operation: a baseband recording mode and a digital filterbank mode

In the baseband recording mode the raw voltages are Nyquist sampled and stored for off-line reduction. The incoming bandwidth can be reduced by applying a Finite Impulse Response (FIR) filter, with reduction factor $2,4, \ldots, 32$. This filter exhibits a flat passband and a rapid fall off. Data can be stored in 1, 2,4 or 8 bits. If no FIR filter is applied and both polarizations are stored in 2 bits, a maximum of 2 bands $\times 10 \mathrm{MHz}$ can be recorded.

The digital filterbank mode performs a Fast Fourier Transform (FFT) on the Nyquist sampled voltages to generate frequency channels $(16,32, \ldots, 4096$ over a $10 \mathrm{MHz}$ band). It calculates the four Stokes parameters (I, Q, U and V) on-line. Consecutive samples can be added to reduce both the time resolution and the data rate. The sampling rate is dependent on the number of channels and ranges from $1.6 \mu \mathrm{s}$ (only possible for 16 channels), $3.2 \mu \mathrm{s}$ ( 16,32 channels) to $2.4576 \mathrm{~ms}$ (all). Samples can be stored in $1,2,4$ or 8 bits. If no samples are added, one Stokes parameter can be recorded in 2 bits for all eight clusters. 